\title{
Advanced Lightweight BFT Process ForTrustworthy Management inWeb Services Computing System
}

\author{
Mr.B.Karthikraja ${ }^{1}$ (M.Tech) Scholar, Mr.K.P.Kaliyamurthie ${ }^{2}$ (H.O.D) \\ Department of Computer Science Engineering, Bharath University-Chennai, Tamilnadu-India.
}

\begin{abstract}
The project entitled as "Trustworthy Coordination of Web Services Atomic Transactions". The consumer will enter their customer id and PIN for accessing their account details. They can view all the accounts across all branches of Net Bank locations online and as well affect fund transfers on real time basis within the Bank network. The accounttransmissions are stored in your database at Net Bank using some services. Such as, Activation service, Registration service, Completion service, Coordinator serviceIn activation services, creates a Coordinator thing and a transaction based context for each transaction. Essentially the Activation service performsidentical factory object that creates Coordinator objects. The Registration service allows the Participants and the Initiator to register their end point references. The Completion service allows the Initiator to signal the start of the distributed commit. The Coordinator service runs the $2 P C$ protocol, which confirms atomic responsibility of the distributed transaction. Information. A View Change algorithm is used to select a new primary when the existing primary is suspected to be Byzantine faulty.
\end{abstract}

Keywords: DES: Data Encryption Standard, AES-Advanced Encryption Standard,BFT-Byzantine Fault Tolerance, WS-AT Web Services Atomic Transaction

\section{Introduction}

Service-oriented computing and web services are transforming The World Wide Web from a publishing Platform into a distributed computing Field, resulting in more and more commercial activities being accompanied Online. Such commercial activities, offered in the open Environment of the Internet, repeatedly involve economic, Healthcare and other web services that their users require being trustworthy. We present a lightweight Byzantine fault tolerance (BFT) process, which can be used to reduce the coordination of web Services business activities (WS-BA) more dependable. The lightweight strategy of the BFTprocess is the result of a comprehensive Study of the threats to the WS-BA coordination services and a careful analysis of the state model of WS-BA. The lightweight BFT

Algorithm uses source ordering, rather than entire ordering, of received requests to realize Byzantine error tolerant, state-machine Replication of the WS-BA coordination services. We have employed the lightweight BFTprocess, and incorporated it into the open source framework, which implements the WS-BA specification with the WS-BA-I extension. Actaevaluation results Obtained from the prototype implementation confirm the efficiency and effectiveness of our lightweight BFTprocess, associated to Old-styleBFT techniques.

\section{Project Scope}

We have incorporated our BFT protocols and mechanisms into an open-source framework that implements the standard WS-AT specification. The enlarged WS-AT framework displays only moderate runtime overhead. It outperforms a reference execution that naively applies the PBFT algorithm to the WS-AT coordination problems, in both LAN and WAN environments. The amplified WS-AT framework is mainly useful for business applications based on transactional Web Services that require a high degree of reliability, safety and trust.

\section{Existing System}

To prevent a faulty primary from hindering the liveness of the Activation protocol or the Completion and Distributed protocol, or distributing conflict information to different replicas, a View Change algorithm is used. A backup replica initiates a view change when it cannot advance to the next phase within a rational time, or when it identifies that the primaryhasIncompatiblestatistics. A View Change procedure is used to choice a new primary when the existing primary is suspected to be Byzantine faulty sent

\subsection{DISADVANTAGE}

- $\quad$ Less Security

- Low performance

- A backup copy initiates a sight change when it cannot advance to the next phase within a reasonable time, or when it detects that the primary has sent conflicting information. 
- A defective primary Coordinator model cannot reuse an obsolete registration or vote to force a transaction outcome against the will of a no faulty Participant.

\section{Proposed System}

This paper is a lightweight BFT framework for trustworthy coordination of Web Services Atomic Transactions that exploits the semantics of the WSAT interactions to achieve better performance than a generalpurpose BFT algorithm that is naively applied. We distinguish that not every process in WS-AT requires Byzantine agreement among the Coordinator models and, thus, that the entireamount of Byzantine agreements needed in a typical transaction can be sharply reduced. More specifically, our BFT framework uses a lightweight protocol instead of running an instance of Byzantine agreement for registration of everyMember. The protocol consumes, at everyMember, the gathering of registering acknowledgments from a quorum of Coordinator replicas, and a round of message interchange at the start of the two-phase commit protocol.

\section{ADVANTAGES:}

a) High protection, safety

b) Safeties' in funds transaction.

c) Good performance.

d) The cost savings are substantial when the number of Participants is large.

e) Reduce the number of Byzantine agreements needed to achieve atomic termination of a Web Services Atomic Transaction.

\section{Architecture Diagram}

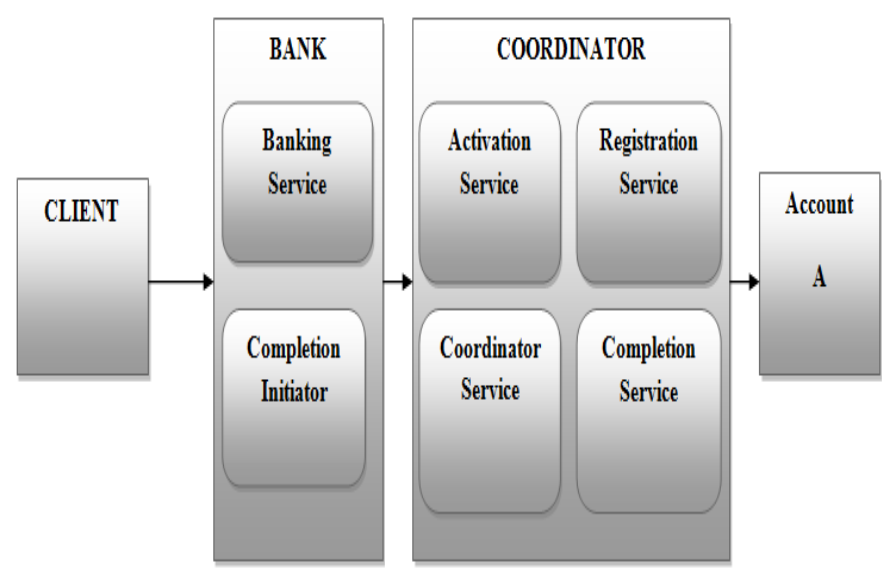

Figure.(1)

\subsection{OVERALL DESCRIPTIONS:}

The internet based Services Atomic business transactioncircumstance makes it possible for businesses to employ in standard distributed transaction processing over the Internet using Web Services technology. For the commercial applications, responsible coordination of WS-AT is essential. In this paper, we illuminate how to render WS-AT coordination trustworthy by applying Byzantine Fault Tolerance (BFT) techniques.

we show how to care for the core services described in the WS-AT configuration, namely, the Activation service, the Registration service, the Completion service and the Coordinator service, against Byzantine faults. The main contribution of this work is that it exploits the semantics of the WS-AT services to minimize the use of Byzantine Agreement (BA), instead of applying BFTsystems naively, which would be excessivelyluxurious.

We have incorporated our BFT protocols and mechanisms into an open source framework that implements the WS-AT description. The resultant BFT framework for WS-AT is useful for business applications that are based on WS-AT and that require a high degree of reliability, safety, and faith. 


\subsection{GANTT CHART}

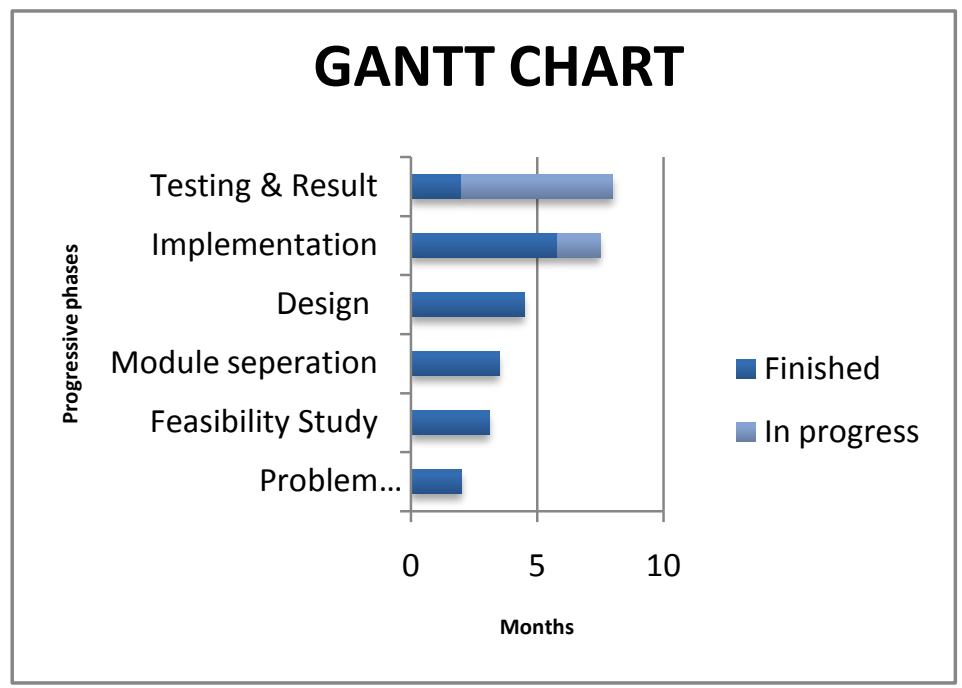

Figure.(2)

\subsubsection{DESIGN SPECIFICATION}

The DFD takes an input-process-output view of a system i.e. data objects the course into the software, are transformed by handlingrudiments, and resultant data objects flow out of the software. Data objects signified by labelled arrows and transformation are represented by circles also called as bubbles.

DFD is presented in a step by step manner i.e. the first data flow model denotes the structure as a complete one. Subsequent DFD improves the context illustration (level 0 DFD), providing cumulative details with each following level. The DFD supports the software engineer to develop replicas of the information domain \& functional domain at the same time.

As the DFD is refined into greater levels of details, the analyst performs an implicit functional decomposition of the system. At the same time, the DFD refinement results in a corresponding refinement of the data as it moves through the process that embodies the applications.

A context-level DFD for the system the primary external entities produce information for use by the system and consume information generated by the system. The labelled arrow represents data objects or object hierarchy.

\section{Rules for DFD:}

- Fix the scope of the system by means of context diagrams.

- Organize the DFD so that the main sequence of the actions

$\circ \quad$ Reads left to right and top to bottom.

$\circ \quad$ Identify entirefeedbacks and yields.

$\circ \quad$ Identify and label each process internal to the system with Rounded circles.

- A process is required for all the data transformation and Allocations. Therefore, never link a data pile to a data Source or the destinations or another data store with just a Data flow arrow.

$\circ \quad$ Do not indicate hardware and ignore control information.

$\circ \quad$ Make sure the names of the processes accurately convey everything the process is done.

- There must not be unidentifiedmethod.Indicate external sources and destinations of the data, with Squares.

- Number each occurrence of repeated external entities.

- $\quad$ Findentire data flows for each coursephase, except simple Record retrievals.

- Label data flow on each arrow.

- Use details flow on each arrow.

- Use the particularstream arrow to specify data activities. 


\subsubsection{DATA FLOW DIAGRAM}

\section{Level 0:}

Level 1:

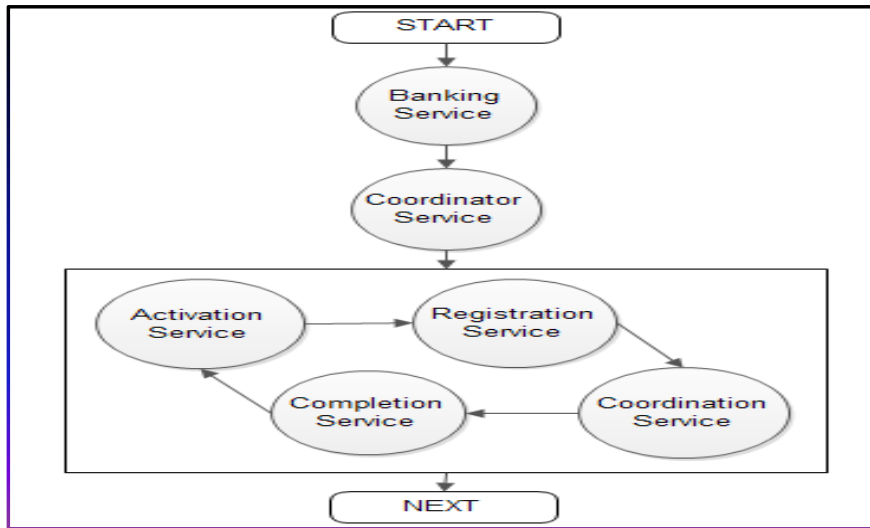

Figure.(3)

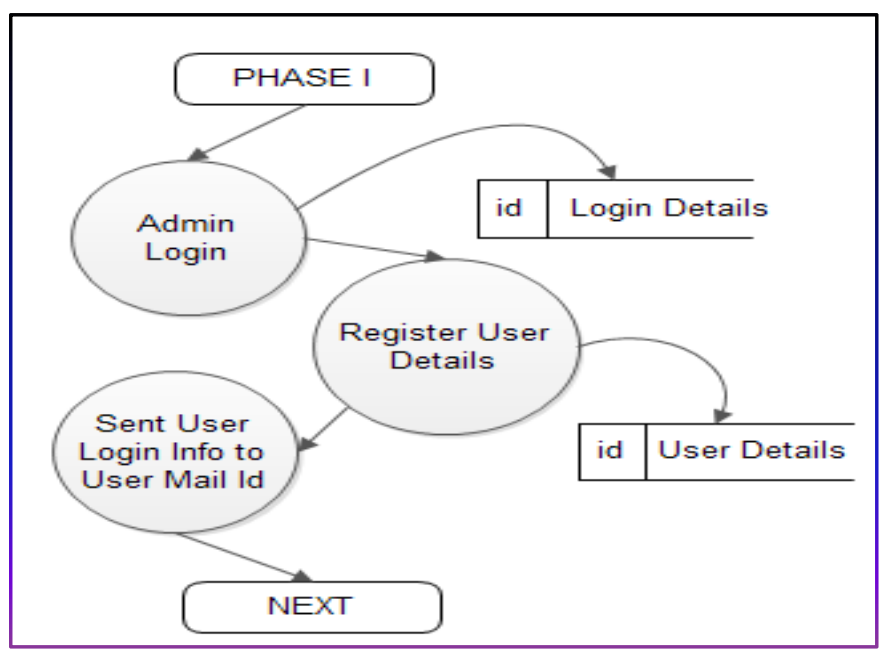

Figure. (4)

Level 2:

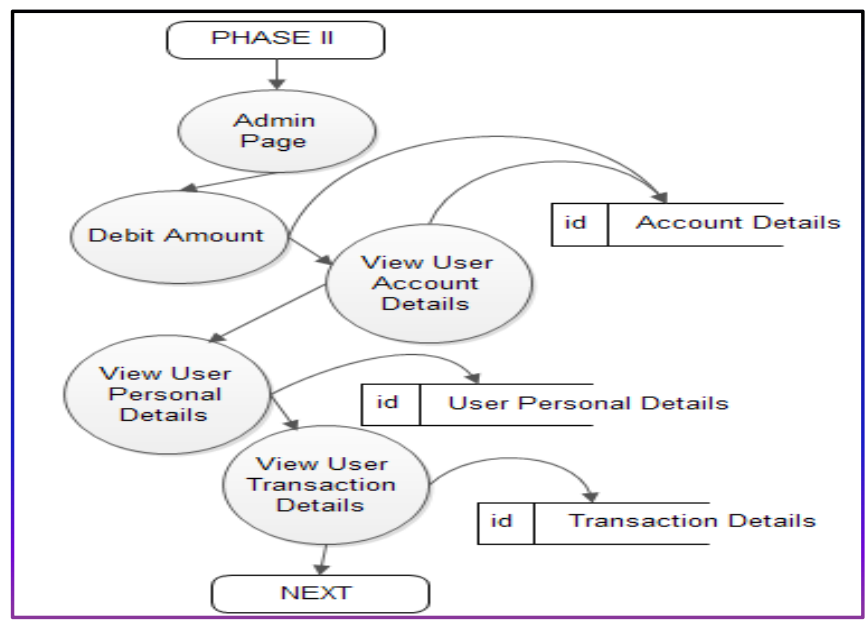

Figure. (5) 
Level 3:

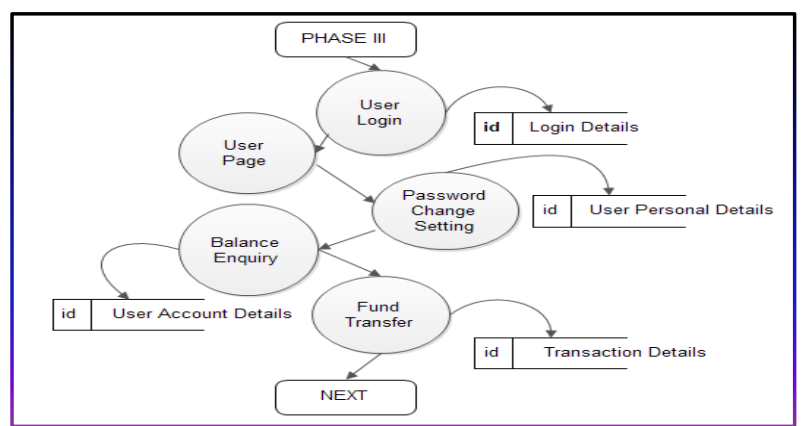

Figure. (6)

Level 4:

5.1 MODULES LIST:

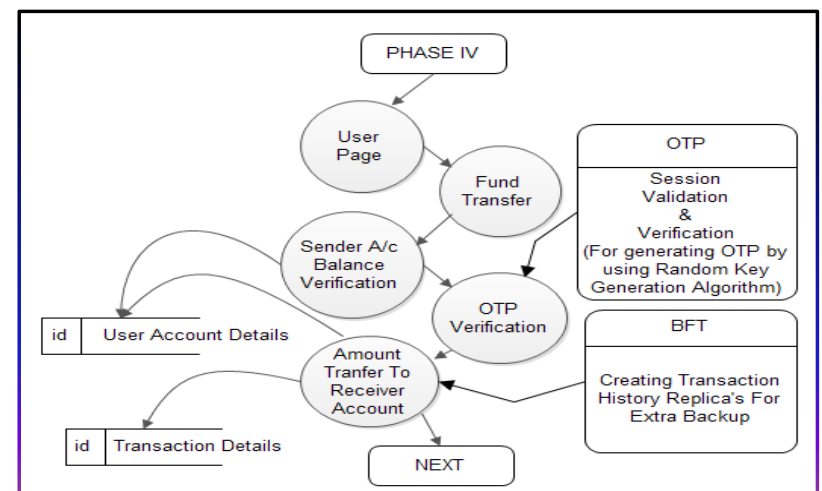

Figure. (7)

V. Modules Descriptions

\begin{tabular}{ll}
$* \quad$ Client Authentication \\
$\quad$ Banking Services \\
$\quad$ Coordinator Services \\
$\quad$ Activation Service \\
$\circ \quad$ Registration Service \\
$\quad$ Coordination Service \\
$\quad$ Completion Service \\
\hline
\end{tabular}

5.2 Modules Discriptions:

Client Authentication:

- Online customers must have access to a computer and a process of expense. In our structure, the handlerinterfaces are login, registration, communication, online payments and operation. Employer details are handled in backend shared database.

- In computer safekeeping, a login or logon is the process by which individual access to a computer system is controlled by identifying and authenticating the user referring to credentials presented by the user.

- A user can log in to a system to obtain access and can then log out or log off when the access is no longer required. To log out is to shut down one's access to a computer system after having previously logged in.

BankingServices:

- Each and every funds transaction process proceeded through the banking services. In banking service contain funds transaction support and verifying authorized person and so on.

Coordinator Services:

- On the Coordinator-side, the services comprise:

a) Activation service: The Activation service creates a Coordinator object and a transaction context for each transaction. Essentially, the Activation service haves like a factory object that creates Coordinator objects.

b) Registration service: The Registration service allows the Participants and the Initiator to register their final point positions. 
c) Coordinator service: The Coordinator service runs the $2 \mathrm{PC}$ protocol, which ensures atomic commitment of the distributed transaction.

d) Completion service: The Completion service allows the originateto signal to start the distributed commend.

Account Services:

- The account service comprises:

a) Completion Initiator service. The Completion Initiator service is used by the Coordinator to inform the Initiator of the final outcome of the operation, as share of the End protocol.

b) Participant service. The Participant service is used by the Coordinator to ask votes from, and to send the operationresult to the Applicant.

\section{Conclusions}

This analysis has enabled us to develop a lightweight BFT algorithm that mitigates the threats and avoids the runtime overhead associated with traditional BFT algorithms. We are going to implement the lightweight BFT algorithm and associated mechanisms, and have incorporated them into the open-source framework that implements the WS-BA specification and the WS-BA-I extension.

The performance evaluation results obtained using the prototype implementation show moderate overhead, especially in a wide-area network, where WS-BA is usuallyorganized. The examination that we have termed in this paper fits in well with the recent trend of cloud computing. Several large organizations, such as Amazon, Google, Microsoft, and Apple, are now offering cloud services.

\section{Author Profile}

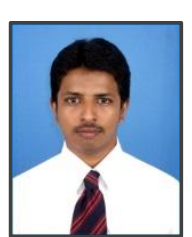

Mr.B.Karthikrajawas born in 1986 in Tamilnadu, India. He was received the B.E. Computer Science Engineering from Jerusalem College of Engineering,Chennai,Tamilnadu, India And presently doing his Master of Technology in Computer science Engineering at Bharath University, Chennai, Tamilnadu, India.

\section{Reference}

[1] The web services-businessactivity-initiator (WS-BA-I) protocol: an extension to the web services-business activity specificationhanneserven, georghicker, christianhuemer and marcozaptletal.

[2] A survey of trust in internet applications - tyronegrandison and morrissloman

[3] Secure and scalable replication in phalanx - dahlia malkhi, michael K. Reiter

[4] Thema: byzantine-fault-tolerant middleware forweb-service applications - michael G. Merideth, aruniyengar, thomasmikalsen, stefan tai, isabellerouvellou, priyanarasimhan

[5] The securegroup group communication system - L. E. Moser, P. M. Melliar-smith, N. Narasimhan

[6] Byzantine fault-tolerant web services for n-tier and service oriented rchitectures. -Sajeeva L. Pallemulleharaldur D. Thorvaldssonkenneth J. Goldman

[7] Design and implementation of a byzantine fault tolerance framework for non-deterministic applications - H. Zhang, W. Zhao, L.E. Moser, P.M. Melliar-smith 